
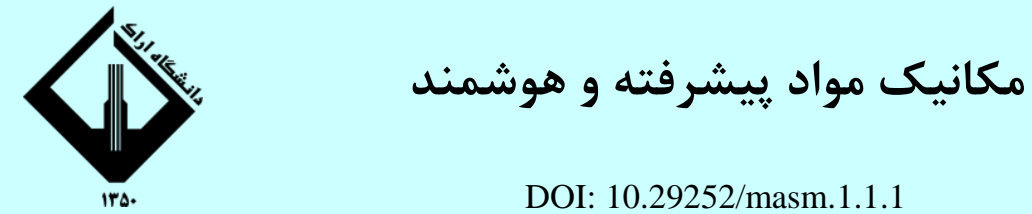

رديابى مسير مشخص ربات متحرك هوشمند بر سطح شيبدار با استفاده از

$$
\text { كنترل كننده غير خطى مد لغزشى }
$$

مصطفى ناظمى زاده الف* ، يويا ملاحى كلاهى ب

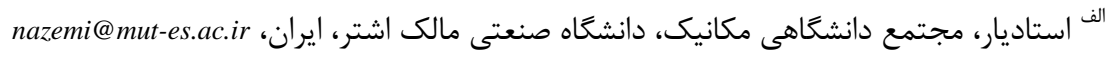

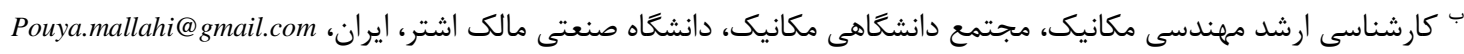

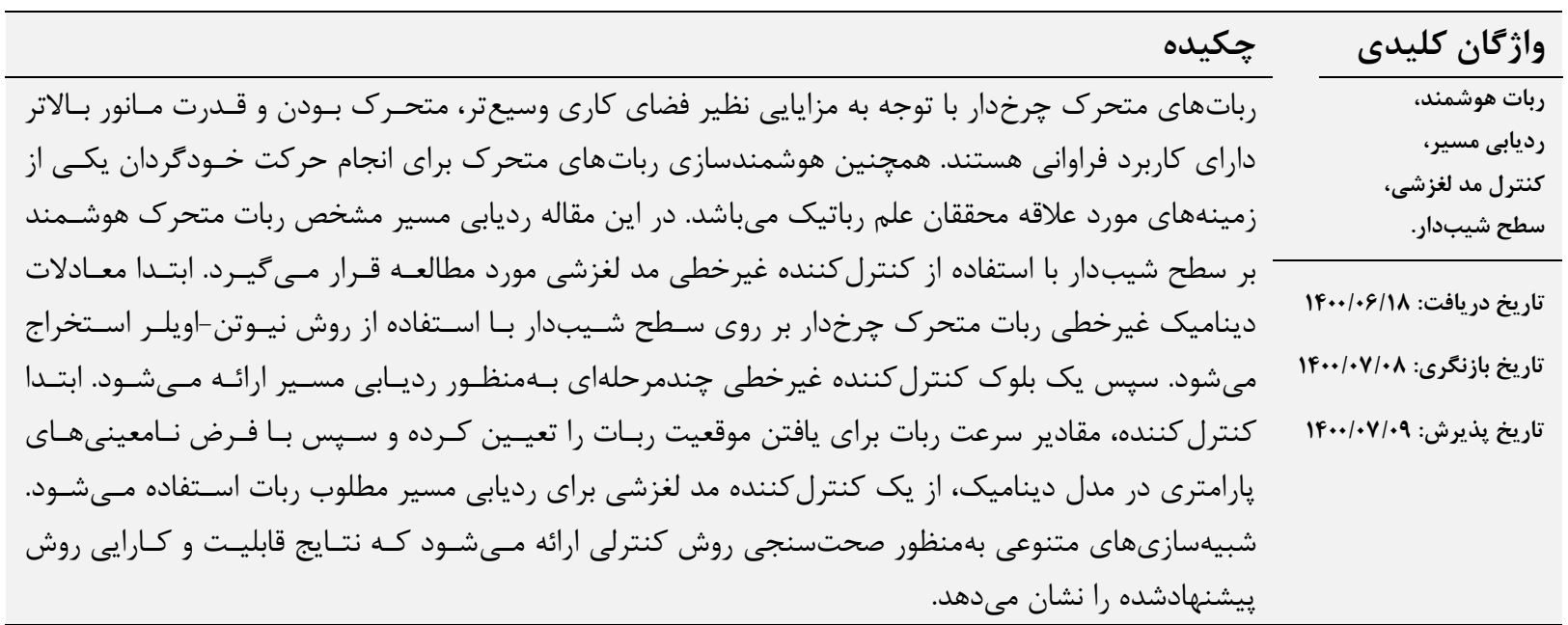

\title{
Trajectory tracking of an intelligent mobile robot on a slope surface using the nonlinear sliding mode control
}

\author{
M. Nazemizadeh ${ }^{a^{*}}$, P. Mallahi kolahi ${ }^{b}$
}

${ }^{a}$ Faculty of Mechanics, Malek-Ashtar University of Technology, Isfahan, Iran

${ }^{b}$ Faculty of Mechanics, Malek-Ashtar University of Technology, Isfahan, Iran

\begin{tabular}{|c|c|}
\hline K E Y W O R D S & A B S T RACT \\
\hline $\begin{array}{l}\text { Intelligence robot, } \\
\text { Trajectory Tracking, } \\
\text { Sliding mode control, } \\
\text { Slope surface. }\end{array}$ & \multirow{4}{*}{$\begin{array}{l}\text { Wheeled mobile robots have many applications due to their advantages such as wide } \\
\text { workspace, mobility and maneuverability. Intelligence of mobile robots to perform } \\
\text { autonomous movements is also one of the favorite fields of robotics researches. In this } \\
\text { paper, the trajectory tracking of an intelligent mobile robot on a sloping surface is studied } \\
\text { using a nonlinear sliding mode control. First, the nonlinear dynamic equations of a wheeled } \\
\text { mobile robot are derived on a sloping surface using the Newton-Euler method. A multistage } \\
\text { nonlinear control block is then proposed for trajectory tracking. First, the controller } \\
\text { calculates the linear and angular velocity of the robot to find the position of the robot, and } \\
\text { then, assuming uncertainties in the dynamic model, a sliding model controller is used to } \\
\text { track the robot's specific path. Various simulations are presented to validate the control } \\
\text { method, which the results show the capability and efficiency of the proposed method. }\end{array}$} \\
\hline Received: September 09, 2021 & \\
\hline Revised: September 30, 2021 & \\
\hline Accepted: October 01, 2021 & \\
\hline
\end{tabular}




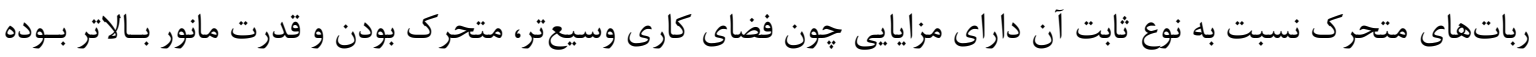

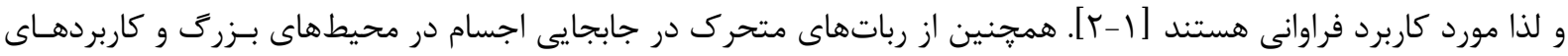

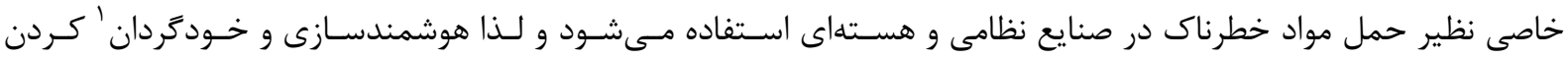

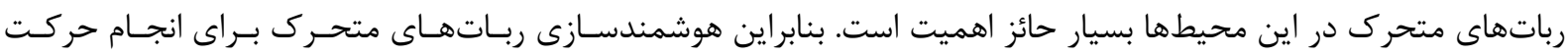

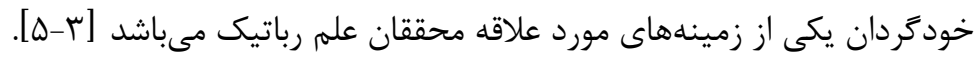

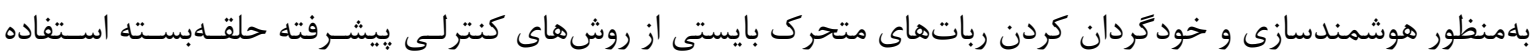

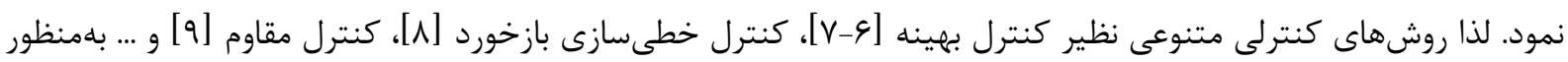
كنترل هوشمند رباتهاى خودَردان توسط محققان علم رباتيك ارائه شده است.

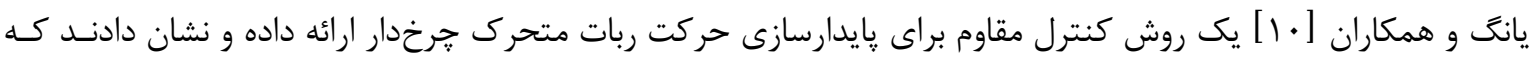

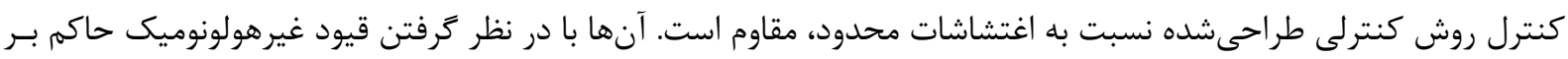

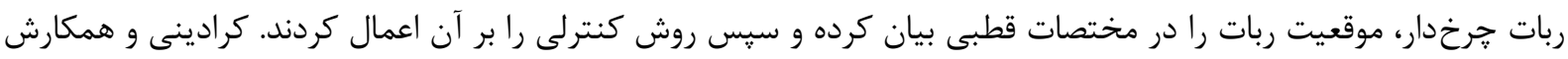

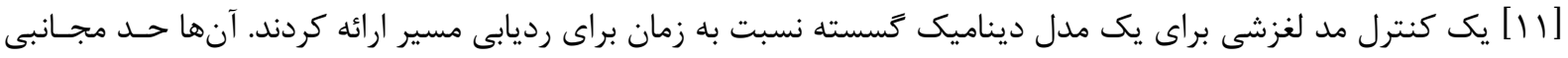

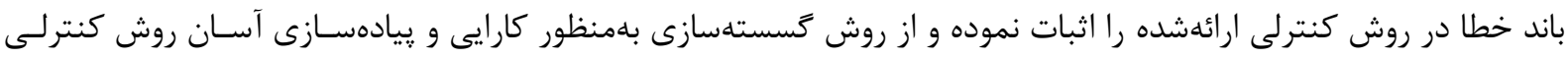

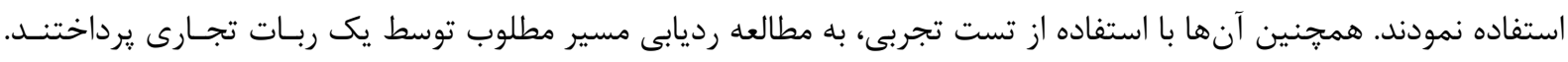

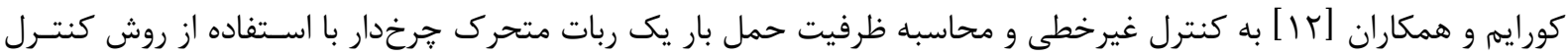

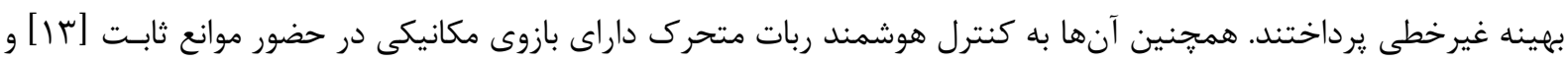

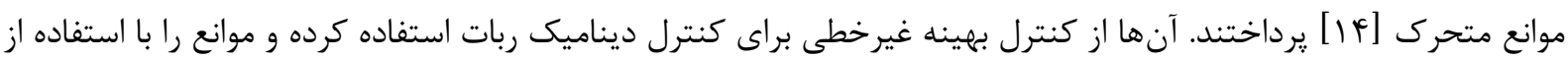

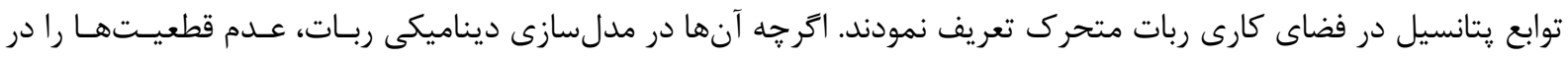

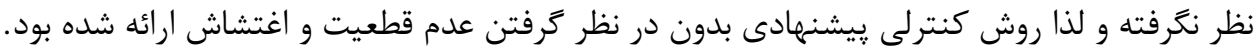

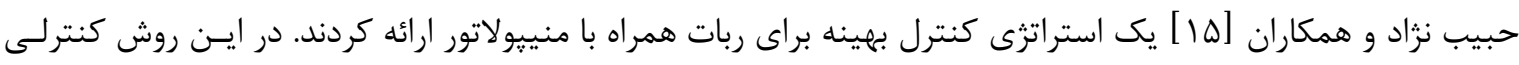

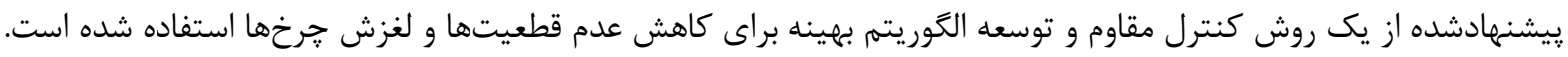

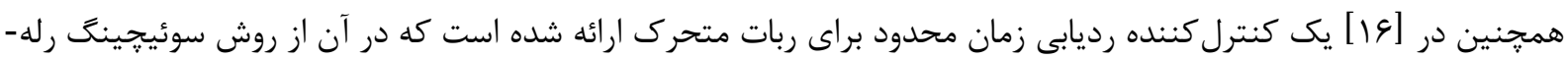

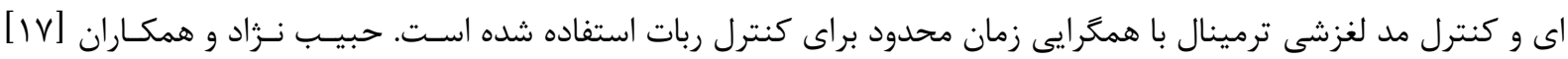

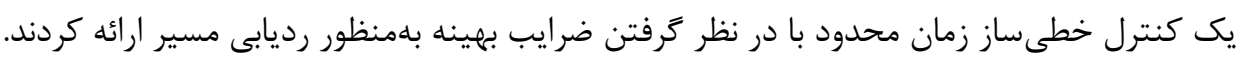

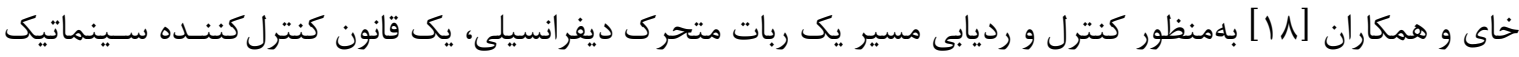
بلهنظور كنترل سرعتهاى ورودى ديناميك ربات ارائه كردند. آنها از كنترل فازى براى افزايش كيفيت رديابى مسير اسـتفاده

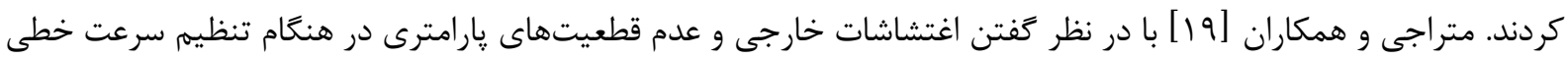

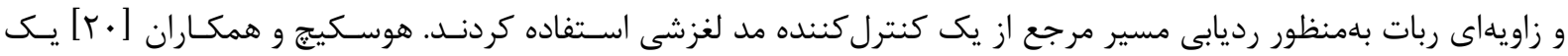

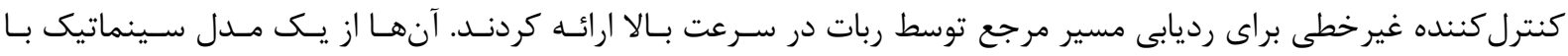

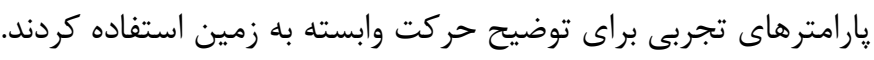

در مقاله كنونى به رديابى مسير ربات متحرك هوشمند بر سـطح شـيبدار همـراه بـا در نظـر گَــفتن نـامعينى دينـاميكى

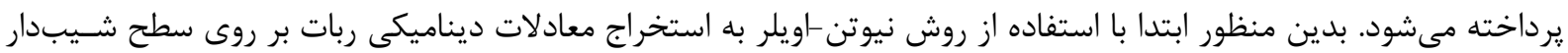

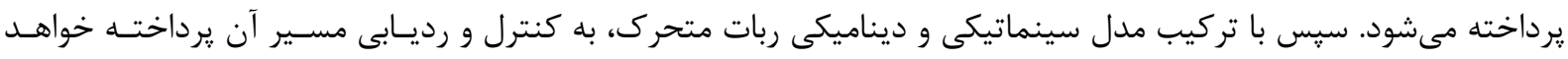

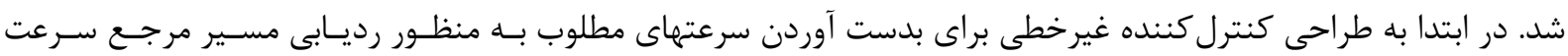

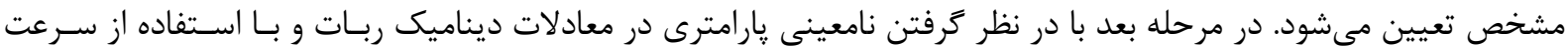

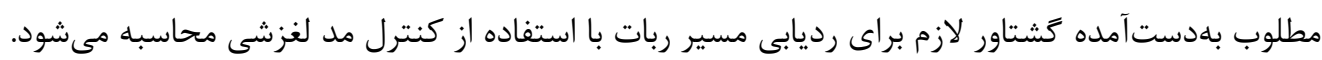




\section{r- استخراج معادلات ديناميكى ربات}

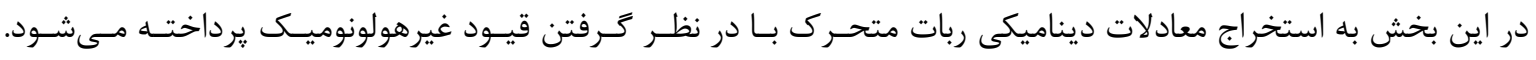

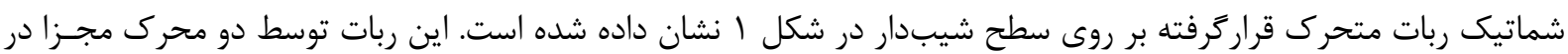
ترخها كنترل مىشود.

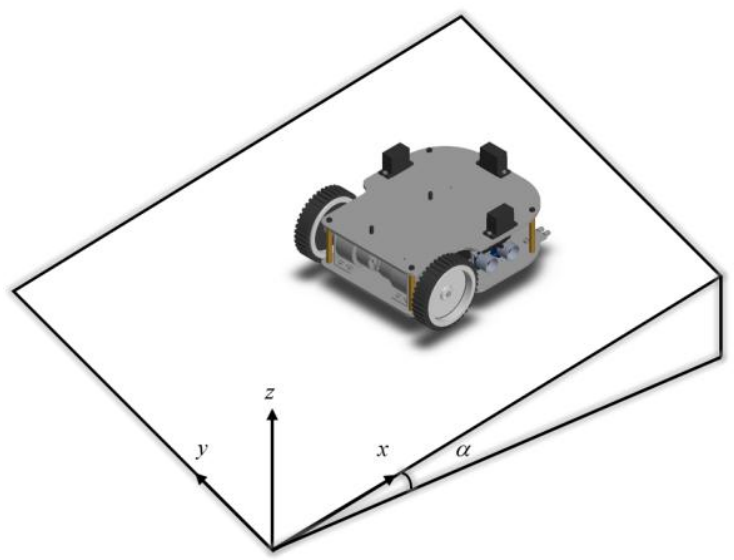

شكل ا شماتيك ربات هرخدار بر روى سطح شيبدار

با استفاده از اصل نيوتن-اويلر به استخراج معادلات ديناميك يرداخته مىشود كه برابر است با:

$\sum F=m a_{x}$

$\sum F=m a_{y}$

$\sum M=I \ddot{\varphi}$

با بسط روابط فوق براى ربات هوشمند بر سطح شيبدار، روابط زير حاصل مىشود:

$m \ddot{x}-\left(F_{r}+F_{l}\right) \operatorname{Cos} \varphi+m g \operatorname{Sin}(\alpha)-\lambda \operatorname{Sin} \varphi=0$

$m \ddot{y}-\left(F_{r}+F_{l}\right) \operatorname{Sin} \varphi+\lambda \operatorname{Cos} \varphi=0$

$I \ddot{\varphi}-\left(F_{r}+F_{l}\right) \frac{L}{2}=0$

با تعريف بردار مختصات

$\dot{y} \operatorname{Cos} \varphi-\dot{x} \operatorname{Sin} \varphi=0$

قيد غيرهولونوميك حاكم بر سيستم در فرم ماتريسى زير بيان مىشود:

$A \dot{\vec{q}}=0$ كه در رابطه بالا A بيانكر قيود غيرهولونوميك است و بهصورت زير تعريف مىشود:

$A=\left[\begin{array}{lll}\operatorname{Cos} \varphi & -\operatorname{Sin} \varphi & 0\end{array}\right]$

فرم كلى معادلات ديناميكى سيستم به فرم نهايى بهصورت زير بيان مىكردد:

$M(\vec{q}) \ddot{\vec{q}}+\vec{G}(\dot{\vec{q}}, \vec{q})=B \vec{\tau}+A \vec{\lambda}$

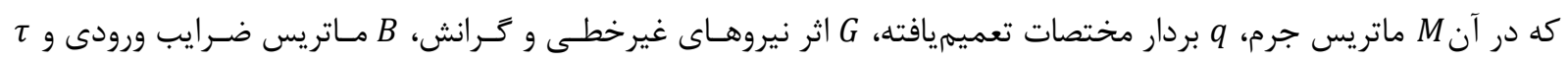

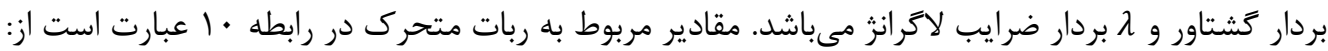

$M=\left[\begin{array}{ccc}m & 0 & 0 \\ 0 & m & 0 \\ 0 & 0 & I\end{array}\right]$

$G=\left[\begin{array}{c}m g \operatorname{Sin}(\alpha) \\ 0 \\ 0\end{array}\right]$ 
$B=\frac{1}{r}\left[\begin{array}{cc}\operatorname{Cos} \varphi & \operatorname{Cos} \varphi \\ \operatorname{Sin} \varphi & \operatorname{Sin} \varphi \\ \frac{L}{2} & -\frac{L}{2}\end{array}\right]$

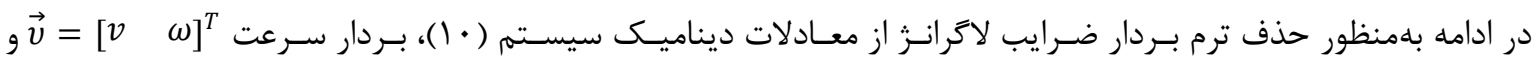

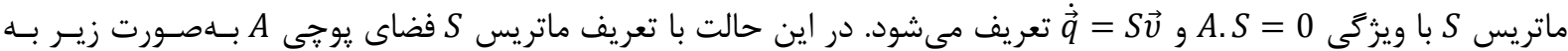

دست مى آيد:

$S=\left[\begin{array}{cc}\operatorname{Cos} \varphi & 0 \\ \operatorname{Sin} \varphi & 0 \\ 0 & 1\end{array}\right]$

معادله ديناميك سيستم با حذف ضرايب لاكرانز بهصورت زير بيان مىشود:

$\overrightarrow{\widetilde{M}}(\vec{q}) \dot{\vec{q}}+\overrightarrow{\widetilde{G}}(\dot{\vec{q}}, \vec{q})=\tilde{B} \vec{\tau}$

مقادير مربوط به رابطه ها باصصورت زير بيان مىشود:

$\overrightarrow{\vec{M}}(\vec{q})=\left[\begin{array}{cc}m & 0 \\ 0 & I\end{array}\right]$

$\overrightarrow{\tilde{G}}(\dot{\vec{q}}, \vec{q})=\left[\begin{array}{c}m g \operatorname{Cos} \varphi \operatorname{Sin} \alpha \\ 0\end{array}\right]$

$\widetilde{B}=\left[\begin{array}{cc}\frac{1}{r} & \frac{1}{r} \\ \frac{L}{2 r} & -\frac{L}{2 r}\end{array}\right]$

r- طراحى كنترل كننده ربات متحرك به روش مد لغزشى

در اين بخش، ساختار كنترل كننده مورد استفاده براى ربات قراركرفته روى سطح شيبدار در شكل r ارائه شده است. ابتدا

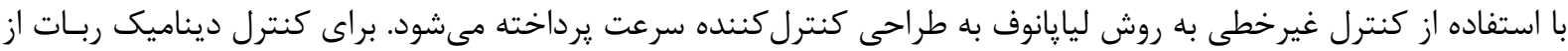

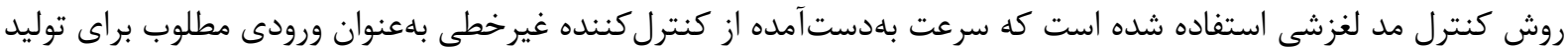

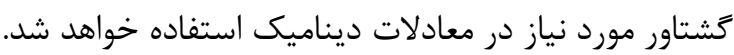

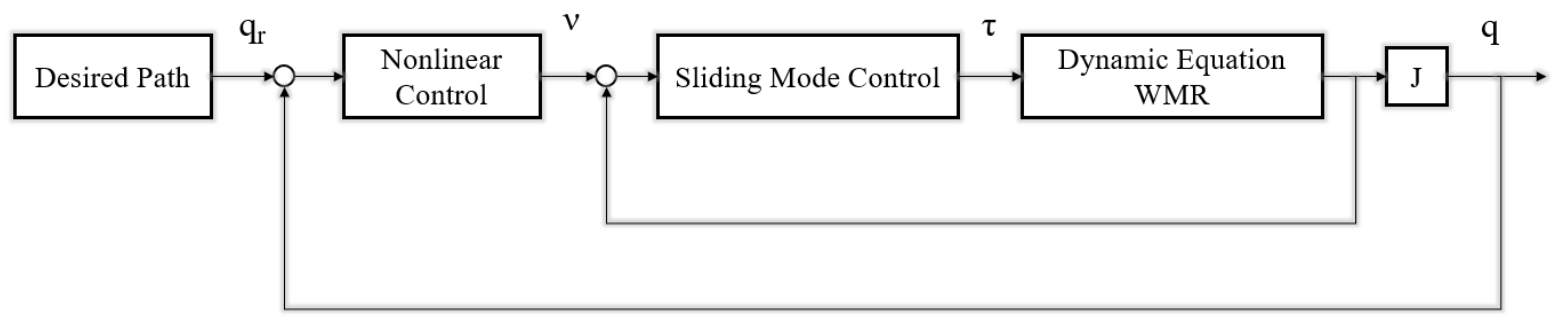

شكل ץ بلوك دياكرام سيستم كنترل غيرخطى ربات متحرى

با تعريف بردار موقعيت ربات

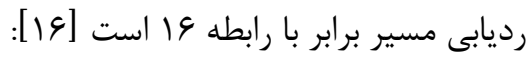

$q_{e}=\left[\begin{array}{l}x_{e} \\ y_{e} \\ \varphi_{e}\end{array}\right]=\left[\begin{array}{ccc}\operatorname{Cos} \varphi & \operatorname{Sin} \varphi & 0 \\ -\operatorname{Sin} \varphi & \operatorname{Cos} \varphi & 0 \\ 0 & 0 & 1\end{array}\right]\left[\begin{array}{l}x_{r}-x_{c} \\ y_{r}-y_{c} \\ \varphi_{r}-\varphi_{c}\end{array}\right]$

با مشتق گيرى از رابطه 19، رابطهى زير به دست مى آيد:

$\dot{q}_{e}=\left[\begin{array}{c}\dot{x}_{e} \\ \dot{y}_{e} \\ \dot{\varphi}_{e}\end{array}\right]=\left[\begin{array}{c}-v+\omega y_{e}+v_{r} \operatorname{Cos} \varphi_{e} \\ -\omega x_{e}+v_{r} \operatorname{Sin} \varphi_{e} \\ -\omega+\omega_{r}\end{array}\right]$

براى به دست آوردن قانون كنترلى مورد نياز براى رديابى مسير مرجع به روش كنترلى غيرخطى، كانديـد ليإِانوف مثبـت 


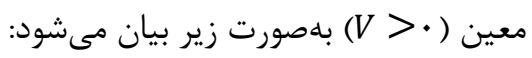

$V=\frac{1}{2}\left(x_{e}^{2}+y_{e}^{2}\right)+\left(1-\operatorname{Cos} \varphi_{e}\right) / K_{y}$

كه در رابطه بالا

$v_{d}=v_{r} \operatorname{Cos} \varphi_{e}+K_{x} x_{e}$

$\omega_{d}=\omega_{r}+v_{r}\left(K_{y} y_{e}+K_{\varphi} \operatorname{Sin} \varphi_{e}\right)$

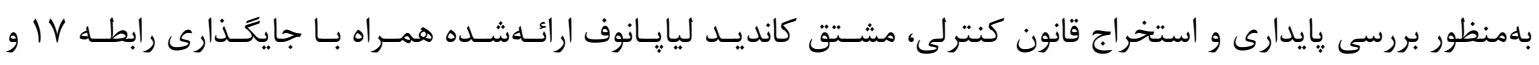
سادهسازى برابر است با:

$$
\begin{array}{r}
\dot{V}=\dot{x}_{e} x_{e}+\dot{y}_{e} y_{e}+\dot{\varphi}_{e} \frac{\operatorname{Sin} \varphi_{e}}{K_{y}} \rightarrow\left[-\left(\omega_{r}+v_{r}\left(K_{y} y_{e}+K_{\varphi} \operatorname{Sin} \varphi_{e}\right)\right) y_{e}-K_{x} x_{e}\right] x_{e}+\cdots \\
{\left[-\left(\omega_{r}+v_{r}\left(K_{y} y_{e}+K_{\varphi} \operatorname{Sin} \varphi_{e}\right)\right) x_{e}+v_{r} \operatorname{Sin} \varphi_{e}\right] y_{e}+\cdots} \\
{\left[-v_{r}\left(K_{y} y_{e}+K_{\varphi} \operatorname{Sin} \varphi_{e}\right)\right] \operatorname{Sin} \varphi_{e} / K_{y} \rightarrow}
\end{array}
$$

$\dot{V}=-K_{x} x_{e}^{2}-\frac{v_{r} K_{\varphi} \operatorname{Sin}^{2} \varphi_{e}}{K_{y}} \leq 0$

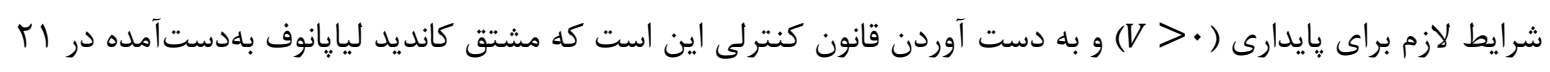

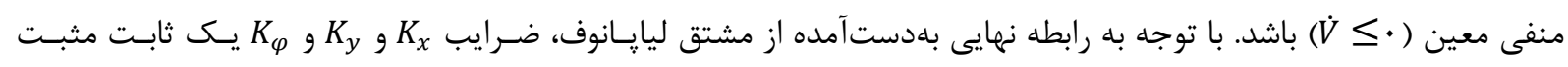

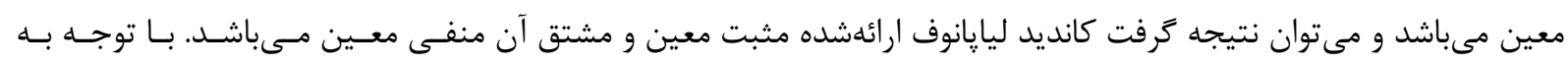

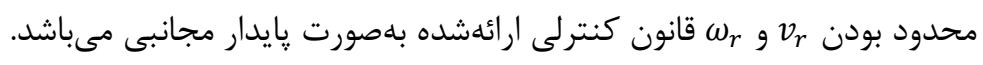

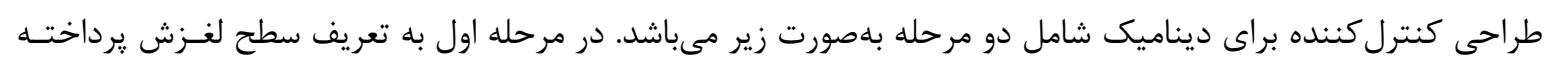

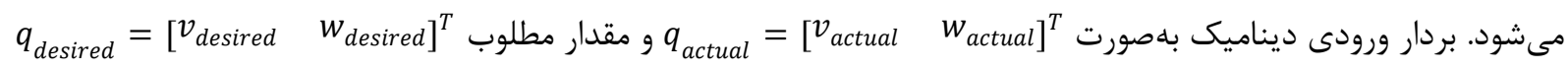

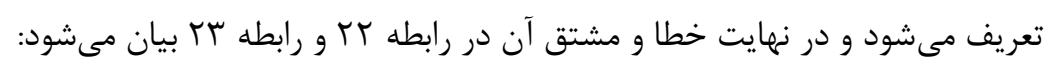

$$
\begin{aligned}
& e=q_{\text {desired }}-q_{\text {actual }} \\
& \dot{e}=\dot{q}_{\text {desired }}-\dot{q}_{\text {actual }}
\end{aligned}
$$

سطح لغزش در نظر گرفتهشده براى كنترل بردارهاى حالت سيستم بهصورت زير بيان مىشود:

$S(t)=\left[\begin{array}{l}s_{1}(t) \\ s_{2}(t)\end{array}\right]=\left[\begin{array}{l}e_{1}(t) \\ e_{2}(t)\end{array}\right]+\left[\begin{array}{l}\beta_{1} \int_{0}^{t} e_{1}(\tau) d \tau \\ \beta_{2} \int_{0}^{t} e_{2}(\tau) d \tau\end{array}\right]$

با مشتق گيرى از سطح لغزش تعريفشده در رابطه FF باصورت زير بيان مىشود:

$\dot{S}(t)=\left[\begin{array}{c}\dot{s}_{1}(t) \\ \dot{s}_{2}(t)\end{array}\right]=\left[\begin{array}{c}\dot{e}_{1}(t) \\ \dot{e}_{2}(t)\end{array}\right]+\left[\begin{array}{l}\beta_{1} e_{1}(t) \\ \beta_{2} e_{2}(t)\end{array}\right]$ با جايكذارى رابطه rr در رابطه ه و استفاده از رابطه (s)

$\dot{q}_{\text {actual }}=\dot{q}_{\text {desired }}+\beta e+\varepsilon \operatorname{sgn}(s)$

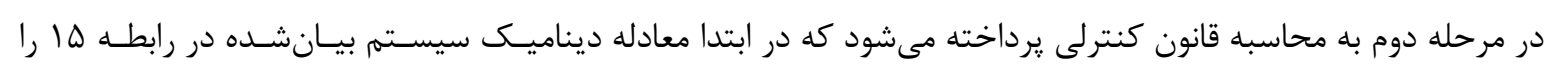
بلهورت زير مىتوان بازنويسى كرد:

$\dot{\vec{q}}=(\overrightarrow{\widetilde{M}}(\vec{q}))^{-1}(\tilde{B} \vec{\tau}-\overrightarrow{\tilde{G}}(\dot{\vec{q}}, \vec{q}))$

با تركيب رابطه צr و رابطه VY قانون كنترل ديناميك برابر با رابطه \Y به دست مىآيد:

$\tau=\widetilde{B}^{-1}\left(\widetilde{M}\left(\dot{q}_{\text {desired }}+\beta e+\varepsilon \operatorname{sgn}(s)\right)+\widetilde{G}\right)$ 


\section{F}

در اين بخش به شبيهسازى ربات هوشمند بر روى سطح شيبدار با استفاده از قانون كنترلى بهدستآمده يرداختـه خواهــد

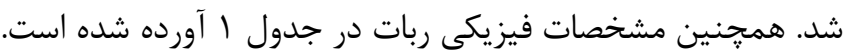

جدول ا. يارامترهاى ربات متحرى

\begin{tabular}{|c|c|c|}
\hline واحد & مقدار & يارامتر ربات \\
\hline $\mathrm{Kg}$ & $m=r$ & جرم ربات \\
\hline $\mathrm{Kg} \cdot \mathrm{m}^{2}$ & $J=r / \Delta$ & ممان اينرسى \\
\hline $\mathrm{m}$ & $r=\cdot / \cdot r$ & شعاع خرخها \\
\hline $\mathrm{m}$ & $b=\cdot / 1 Q$ & فاصله گرخها تا مركز ربات \\
\hline deg & $r$. & زاويه سطح شيبدار \\
\hline
\end{tabular}

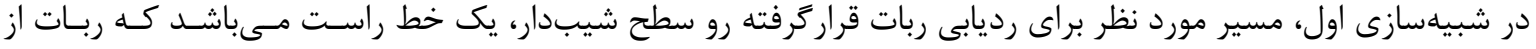
موقعيت دم ${ }^{T}$ مشخص مستقيم در شكل ب نشان داده شده است.

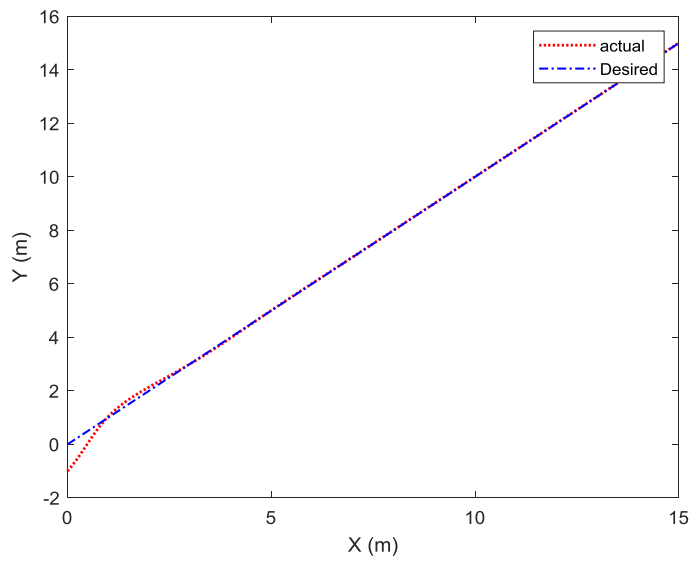

شكل ץ مقايسه مسير ييمودهشده توسط ربات در مقايسه با مسير مرجع مستقيم

همانطور كه در شكل بالا ديده مى شود، موقعيت اوليه ربات از مسير مطلوب به علت شرايط اوليه انتخابى فاصله دارد. اما با استفاده از كنترل كر مد لغزشى، ربات در كمترين زمان به رديابى مسير مرجع يرداخته است. در ادامه خطاى رديـابى مختصـات تعميميافته مختلف ربات نسبت به زمان برابر در شكل \&ٔ نشان داده شده است.

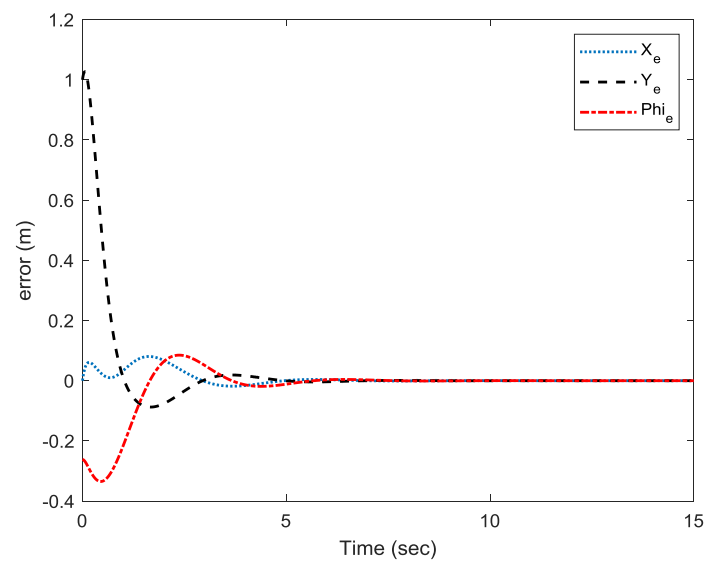

شكل \& خطاى رديابى موقعيت و جهت 
با توجه به نمودار خطا بهدستآمده، يس از كذشت ه ثانيه از زمان حركت، خطاى رديابى مسير مرجع به سمت صـفر ميـل

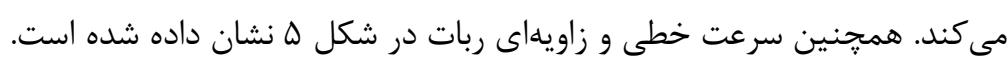
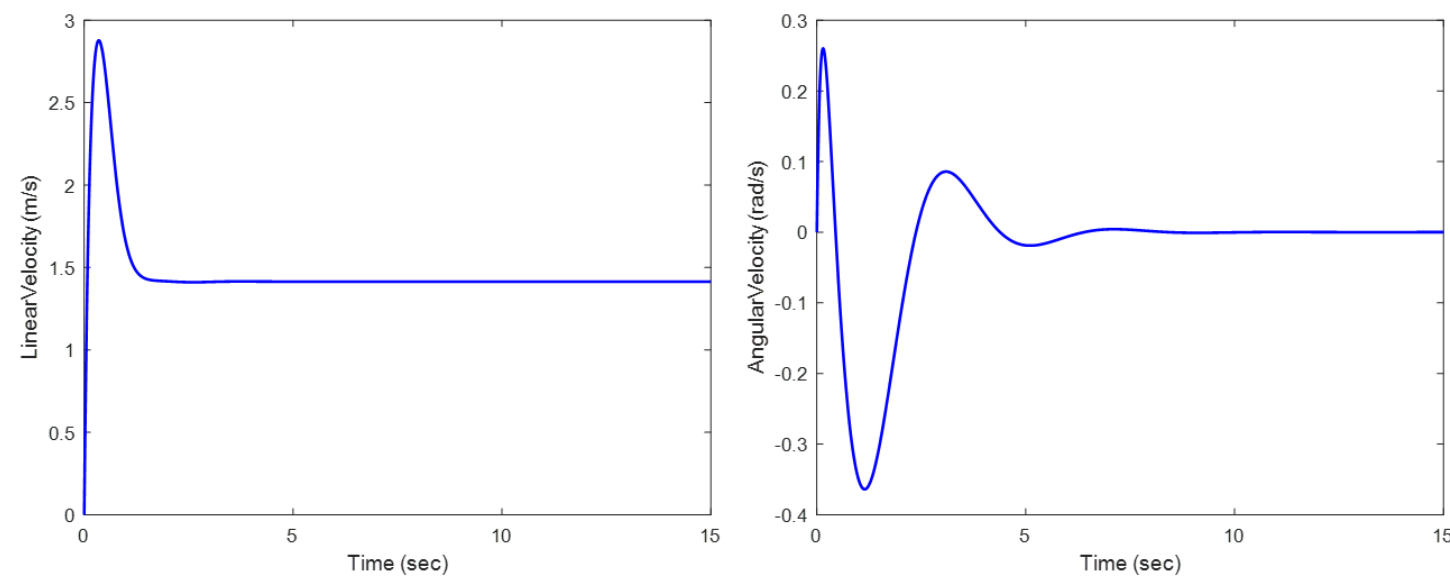

شكل ه سرعت خطى و زاويهاى ربات براى رديابى مسير مرجع مستقيم

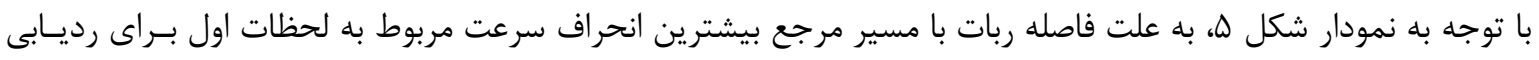

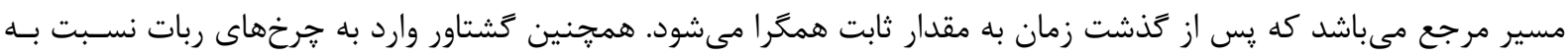
زمان برابر است با شكل وجع.

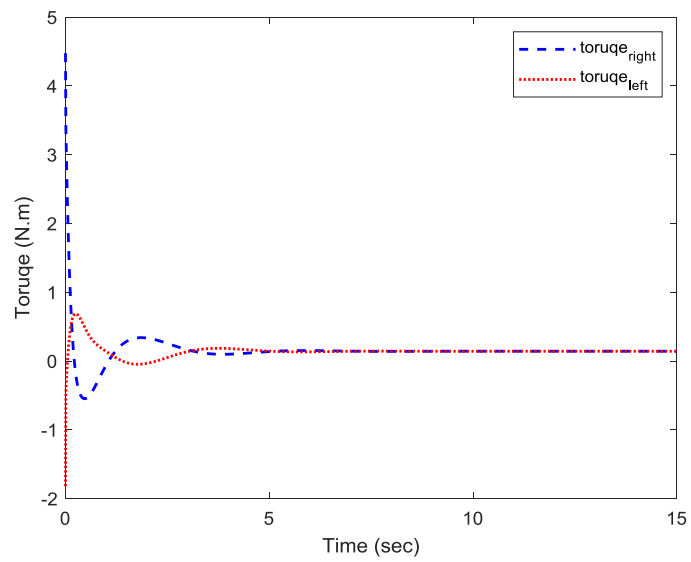

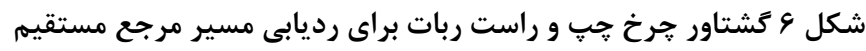

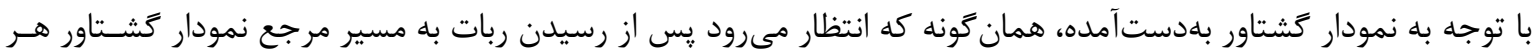

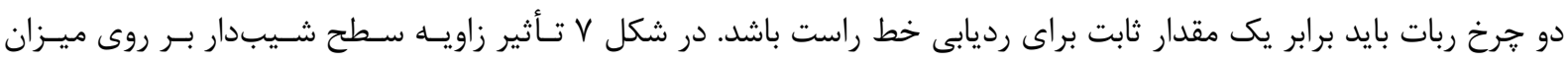
كشتاور لازم براى رديابى مسير نشان داده شده است.

با توجه به نمودار گشتاور بلهدستآمده براى شيبهاى مختلف، همان كونه كه انتظار مىرود، با افزايش زاويه سطح شيبدار،

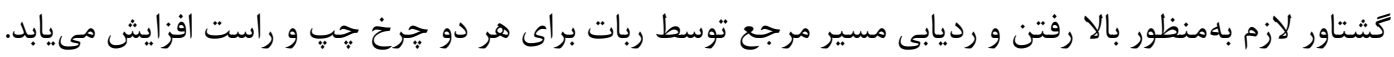
در شبيهسازى دوم، مسير مورد نظر براى رديابى ربات قراركرفته رو سطح شيبدار، يك خط راست مسىباشـد كـهـ ربـات از موقعيت مرجع در شكل ^ نشان داده شده است. 
همانطور كه در شكل ^ مشاهده مىشود، ربات توانيى رديابى مسير يِيجيده سينوسى را نيز دارد. از طرفى سرعت خطى ربات در شكل 9 ارائه شده است.
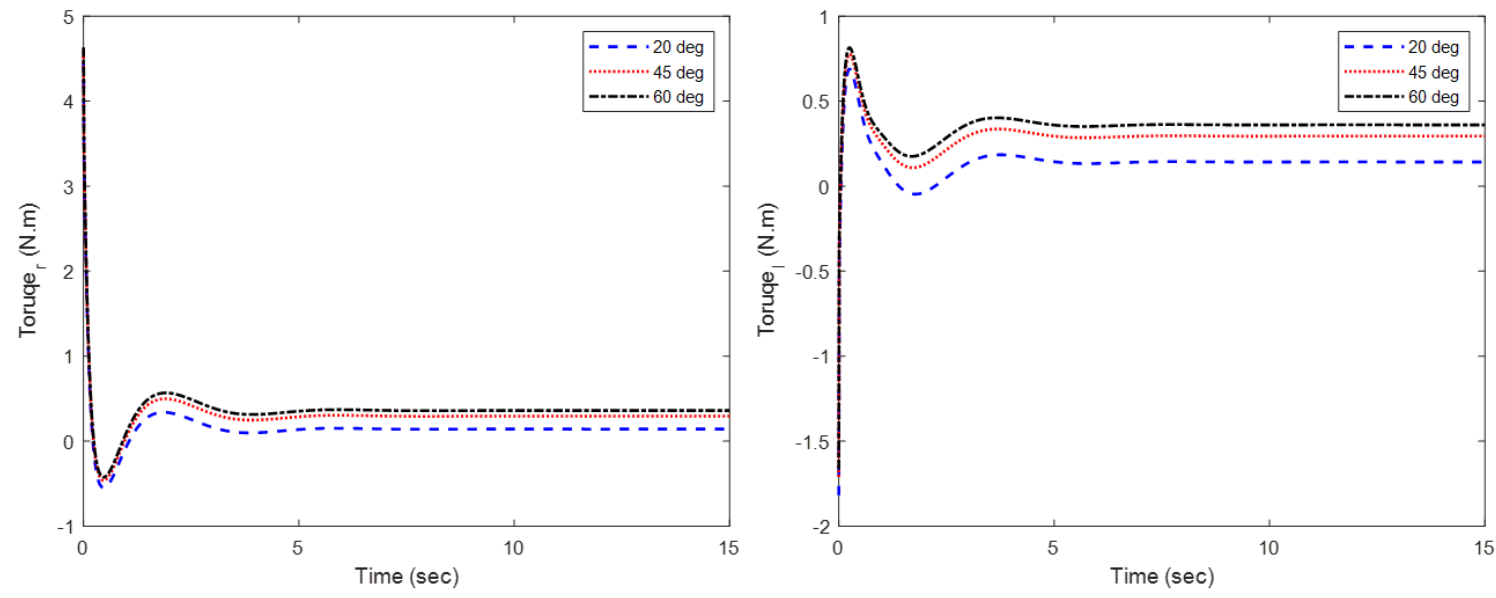

شكل \ تأثير زاويه سطح شيبدار بر روى گشتاور خرخ جٍ و راست براى رديابى مسير مرجع مستقيم

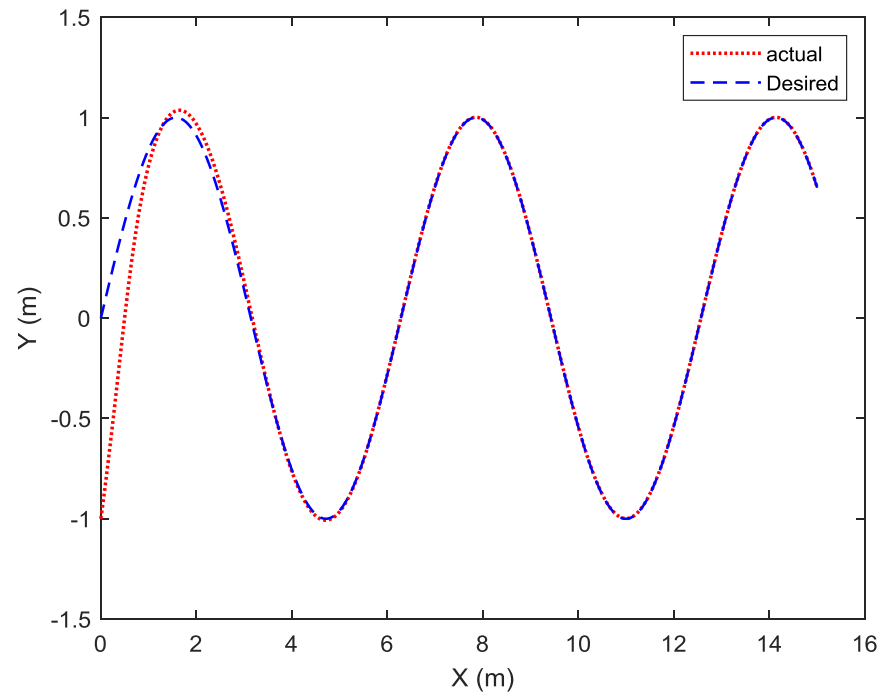

شكل ^ مقايسه مسير يِيمودهده توسط ربات در مقايسه با مسير مرجع سينوسى
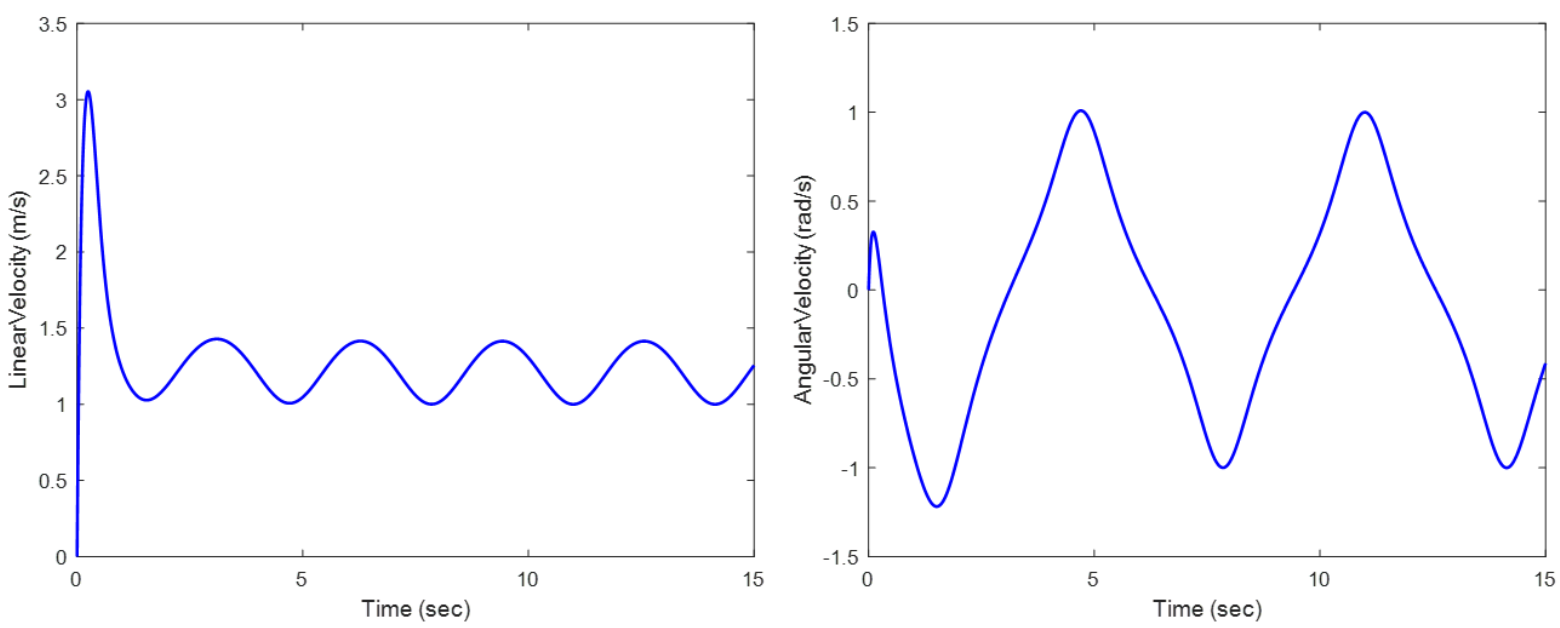

شكل 9 سرعت خطى و زاويهاى ربات براى رديابى مسير مرجع سينوسى 


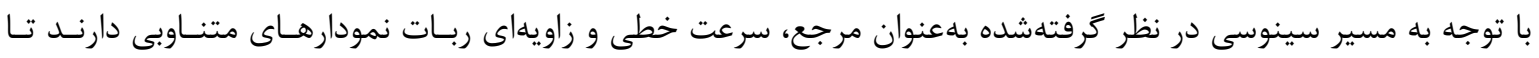
رديابى مسير مرجع بلصورت كامل صورت كيرد. در ادامه گشتاور جرخهاى ربات براى رديابى مسير مرجع در شكل · ا نشان داده شده است.

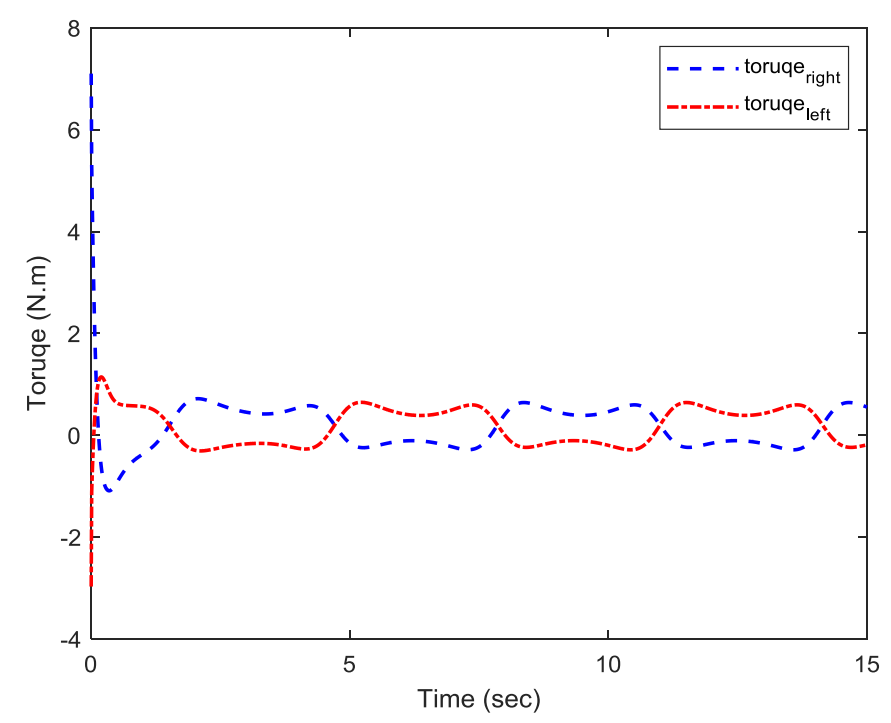

شكل •ا كشتاور خرخ جِ و راست ربات براى رديابى مسير مرجع مستقيم

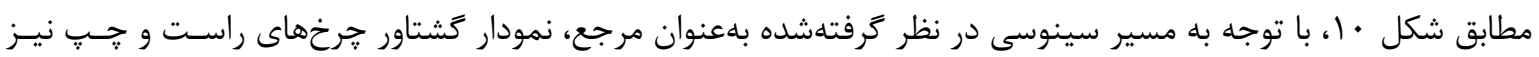

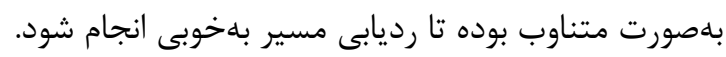
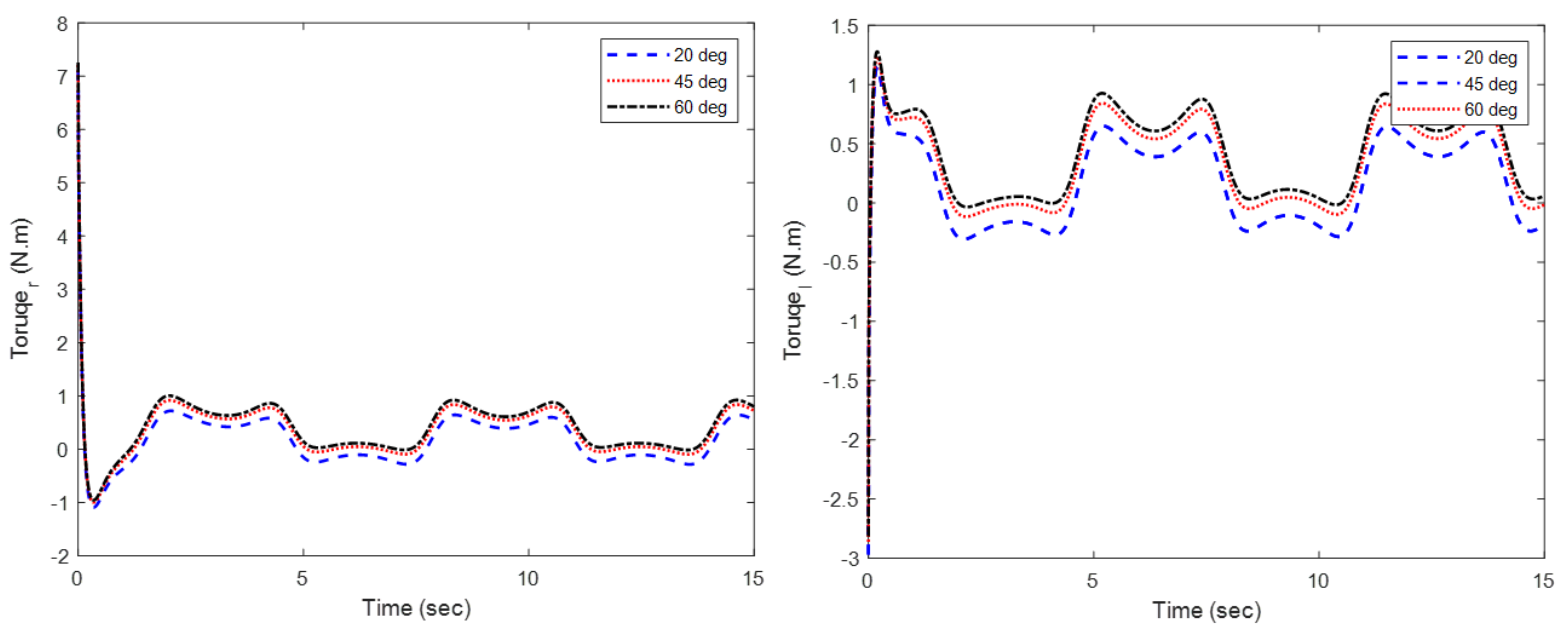

شكل Iا تأثير زاويه سطح شيبدار بر روى كشتاور خرخ جٍ و راست براى رديابى مسير مرجع سينوسى (ع)

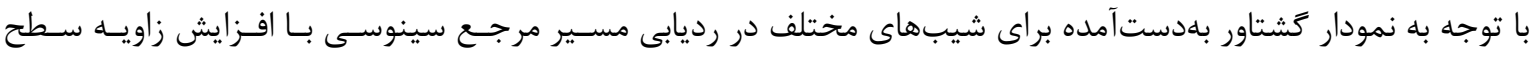

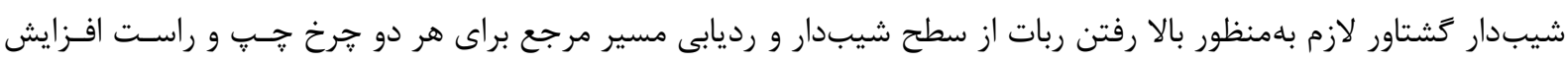
مىيابد.

\section{ه- نتيجه}

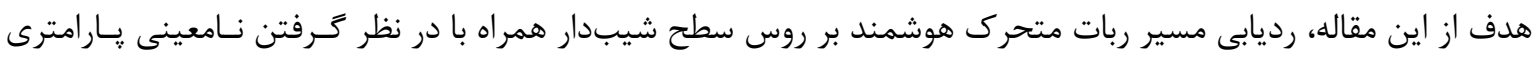

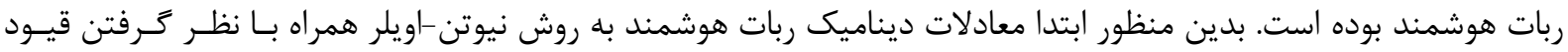

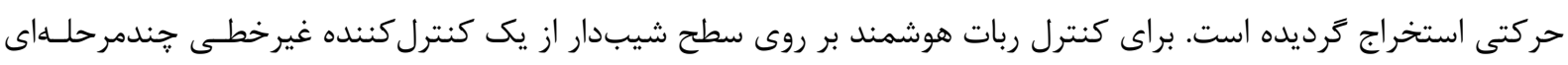




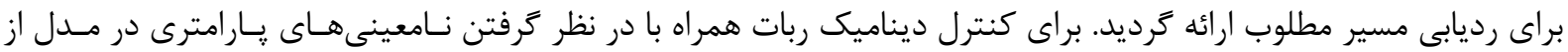

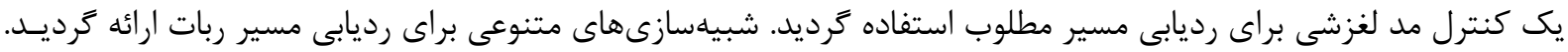

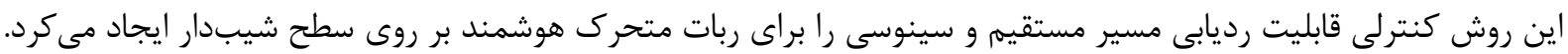

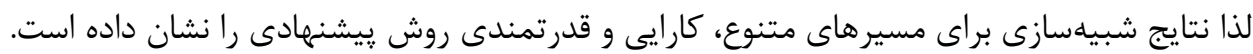

$$
\begin{aligned}
& \text { 4- مراجع }
\end{aligned}
$$

[1] Ben Jabeur C, Seddik H. Design of a PID optimized neural networks and PD fuzzy logic controllers for a two-wheeled mobile robot. Asian Journal of Control. 2021;23:23-41.

[2] Das M S, Samanta A, Sanyal S, Mandal S. Support Value-Based NFSMC for Wheeled Mobile Robot Path Tracking in Unknown Environments. Wireless Personal Communications. 2021;1-21.

[3] Li W, Li Z, Liu Y, Ding L, Wang J, Gao H, Deng Z. Semi-autonomous bilateral teleoperation of six-wheeled mobile robot on soft terrains. Mechanical Systems and Signal Processing. 2019;133:106234.

[4] Gao X, Gao R, Liang P, Zhang Q, Deng R, Zhu W. A hybrid tracking control strategy for nonholonomic wheeled mobile robot incorporating deep reinforcement learning approach. IEEE Access. 2021;9:1559215602 .

[5] Nazemizadeh M, Rahimi H N, Khoiy K A. Trajectory planning of mobile robots using indirect solution of optimal control method in generalized point-to-point task. Frontiers of Mechanical Engineering. 2012;7:2328.

[6] Korayem M H, Nekoo S R, Korayem A H. Finite time SDRE control design for mobile robots with differential wheels. Journal of Mechanical Science and Technology. 2016;30:4353-4361.

[7] Korayem M H, Nazemizadeh M, Nohooji H R. Smooth jerk-bounded optimal path planning of tricycle wheeled mobile manipulators in the presence of environmental obstacles. International Journal of Advanced Robotic Systems. 2012;9:105.

[8] Korayem M H, Yousefzadeh M, Manteghi S. Dynamics and input-output feedback linearization control of a wheeled mobile cable-driven parallel robot. Multibody System Dynamics. 2017;40:55-73.

[9] Roy S, Nandy S, Kar I N, Ray R, Shome S N. Robust control of nonholonomic wheeled mobile robot with past information: Theory and experiment. Proceedings of the Institution of Mechanical Engineers, Part I: Journal of Systems and Control Engineering. 2017;231:178-188.

[10] Yang J M, Kim J H. Sliding mode control for trajectory tracking of nonholonomic wheeled mobile robots. IEEE Transactions on robotics and automation. 1999;15:578-587.

[11] Corradini M L, Orlando. Control of mobile robots with uncertainties in the dynamical model: a discrete time sliding mode approach with experimental results. Control Engineering Practice. 2002;10:23-34

[12] Korayem M H, Rahimi H N, Nikoobin A, Nazemizadeh M. Maximum allowable dynamic payload for flexible mobile robotic manipulators. Latin American applied research. 2013;43:29-35.

[13] Korayem M H, Nazemizadeh M, Nohooji H R. Optimal point-to-point motion planning of non-holonomic mobile robots in the presence of multiple obstacles. Journal of the Brazilian Society of Mechanical Sciences and Engineering. 2014;36:221-232.

[14] Korayem M H, Nazemizadeh M, Rahimi H N. Trajectory optimization of nonholonomic mobile manipulators departing to a moving target amidst moving obstacles. Acta Mechanica. 2013;224:995-1008.

[15] Korayem M H, Ghobadi N, Fathollahi Dehkordi S. Designing an optimal control strategy for a mobile manipulator and its application by considering the effect of uncertainties and wheel slipping. Optimal Control Applications and Methods. 2021;42:1487-1511.

[16] Hosseini M I, Jafari Harandi M R, Khalilpour Seyedi S A, Taghirad H. Experimental performance of adaptive fast terminal sliding mode control on a suspended cable robot. Journal of Electrical and Computer Engineering Innovations (JECEI). 2018;7:59-67.

[17] Korayem M H, Nekoo S R, Kazemi S. Finite-time feedback linearization (FTFL) controller considering optimal gains on mobile mechanical manipulators. Journal of Intelligent \& Robotic Systems. 2019;94:727744. 
[18] Khai T Q, Ryoo Y J, Gill W R, Im D Y. Design of kinematic controller based on parameter tuning by fuzzy inference system for trajectory tracking of differential-drive mobile robot. International Journal of Fuzzy Systems. 2020; 22: 1972-1978.

[19] Matraji I, Al-Durra A, Haryono A, Al-Wahedi K, Abou-Khousa M. Trajectory tracking control of skidsteered mobile robot based on adaptive second order sliding mode control. Control Engineering Practice. 2018; 72: 167-176.

[20] Huskić G, Buck S, Herrb M, Lacroix S, Zell A. High-speed path following control of skid-steered vehicles. The International Journal of Robotics Research. 2019; 38(9): 1124-1148. 